Supporting Information for

\title{
Thermal Transport across Surfactant Layers on Gold Nanorods
}

\section{in Aqueous Solution}

Xuewang Wu,${ }^{\dagger}$ Yuxiang Ni, ${ }^{\dagger}$ Jie Zhu, ${ }^{\dagger}$ Nathan D. Burrows,${ }^{\ddagger}$ Catherine J. Murphy, ${ }^{\ddagger}$ Traian

Dumitrica, ${ }^{\dagger}$ Xiaojia Wang,,${ }^{*} \dagger$

${ }^{\dagger}$ Department of Mechanical Engineering, University of Minnesota, Twin Cities, Minneapolis, MN 55455, USA

\$ Department of Chemistry, University of Illinois at Urbana Champaign, IL 61801, USA

${ }^{*}$ Corresponding Author:

Xiaojia Wang

Email: wang4940@umn.edu 


\section{S1. Gold Nanorod (GNR) Synthesis}

GNRs were synthesized with the aqueous silver-assisted seed-mediated method. Gold seeds were nucleated by mixing $0.25 \mathrm{~mL}$ of $0.01 \mathrm{M} \mathrm{HAuCl}_{4} \cdot 3 \mathrm{H}_{2} \mathrm{O}$ solution and $9.75 \mathrm{~mL}$ of $0.1 \mathrm{M}$ cetyltrimethylammonium bromide $(\mathrm{CTAB})$ aqueous solution, and then adding $0.6 \mathrm{~mL}$ of $0.01 \mathrm{M}$ ice-cold $\mathrm{NaBH}_{4}$ solution. The seeds were allowed to rest for 1 hour before use. To grow the gold nanorods, $9.5 \mathrm{~mL}$ of $0.1 \mathrm{M} \mathrm{CTAB}, 0.5 \mathrm{~mL}$ of $0.01 \mathrm{M} \mathrm{HAuCl}_{4}$, and $100 \mu \mathrm{L}$ of $0.01 \mathrm{M} \mathrm{AgNO}_{3}$ were mixed together. Then $55 \mu \mathrm{L}$ of $0.1 \mathrm{M}$ ascorbic acid were added into the mixture to act as a reducing agent. Next, $12 \mu \mathrm{L}$ of gold seed solution was added and then left undisturbed for 1 hour. Finally, the GNR solution was purified by centrifugation at $11 \mathrm{k}$ rcf and removing the excess surfactants and reactants via decanting and suspending the pellet in $5 \mathrm{mM} \mathrm{CTAB}$ at total of five times. The concentration of GNRs was $2.75 \mathrm{nM}$, as determined by Visible Near-IR Spectroscopy, ${ }^{1}$ and the concentration of CTAB was $5 \mathrm{mM}$, as prepared by multiple washings with a 5 mM CTAB solution, for all transient absorption measurements.

\section{S2. Determination of the Surfactant Thickness}

The hydrodynamic diameters of GNRs with both surfactants obtained from DLS measurements were used to derive the thickness of the surfactants $\left(h_{\mathrm{s}}\right)$, based on the following equations: ${ }^{2-3}$

$$
\begin{gathered}
d_{\mathrm{H}}=\frac{k_{\mathrm{B}} T}{3 \pi \eta D_{\mathrm{t}}}, \\
D_{\mathrm{t}}=\frac{k_{\mathrm{B}} T\left(\operatorname{In}\left(L / d_{\mathrm{cs}}\right)+C_{\mathrm{t}}\right)}{3 \pi \eta L},
\end{gathered}
$$




$$
\begin{gathered}
C_{\mathrm{t}}=0.312+0.565\left(d_{\mathrm{cs}} / L\right)-0.100\left(d_{\mathrm{cs}} / L\right)^{2}, \\
L=L_{\mathrm{GNR}}+2 h_{\mathrm{s}} \\
d_{\mathrm{cs}}=d_{\mathrm{GNR}}+2 h_{\mathrm{s}}
\end{gathered}
$$

where $d_{\mathrm{H}}$ is the hydrodynamic diameter measured from DLS, $k_{\mathrm{B}}$ is the Boltzmann constant, $T$ is the temperature of sample solution (room temperature is used for DLS measurement), $D_{\mathrm{t}}$ is the translation diffusion coefficient, $\eta$ is the solution viscosity, $L_{\mathrm{GNR}}$ and $d_{\mathrm{GNR}}$ are the average length and diameter of bare GNRs obtained from TEM, and $L$ and $d_{\mathrm{cs}}$ are the average total length and total diameter of GNRs coated with surfactants.

\section{S3. Wavelength Selection for Pump and Probe Beams}

In our pump-probe setup, both pump and probe were set at the plasmonic wavelength for each GNR/surfactant/water sample in the transient absorption measurement.

On the one hand, sufficient pump heating of GNR can be achieved with the same input laser power when optical excitation (pump beam) is at the GNR plasmonic wavelength. This can be readily seen from Figure S1, which compares the absorption spectra of the GNR/CTAB sample in aqueous solution (red line) and the surrounding medium of water only (blue line). At the plasmonic resonant wavelength (denoted by the red dashed line), most laser energy will be absorbed by the GNRs rather than the surrounding medium. 


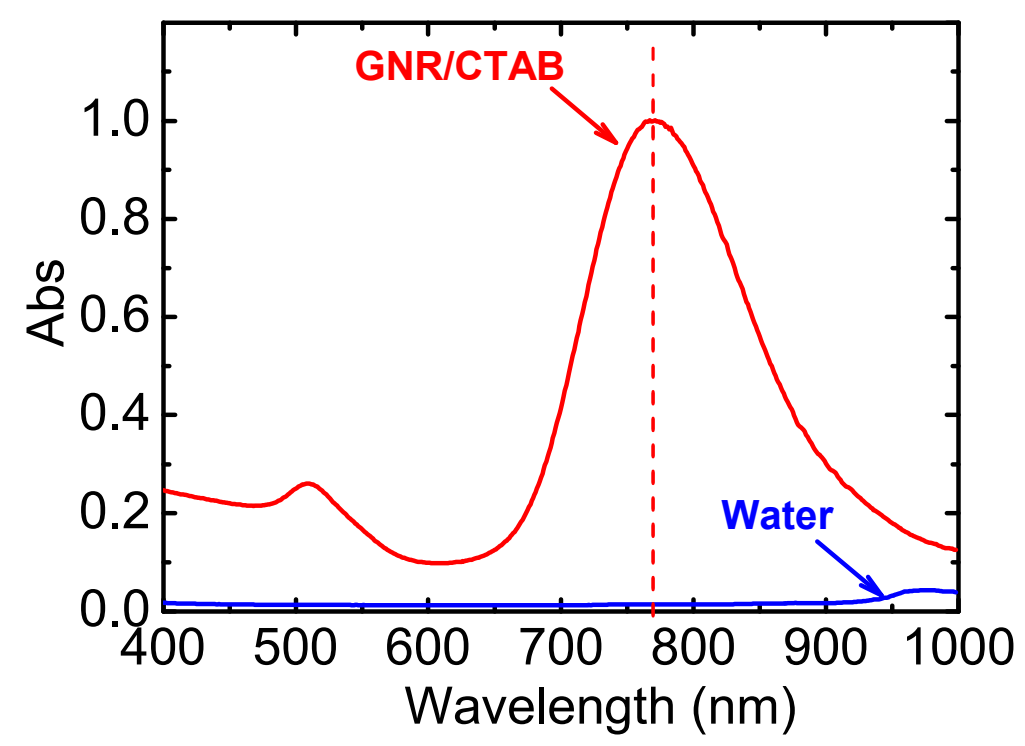

Figure S1. UV-VIS absorption spectra of the GNR/CTAB sample in water (red line) and the surrounding medium of water only (blue line). Both plots are normalized with the plasmonic peak absorption of the GNR/CTAB samples in water solution $(771 \mathrm{~nm})$.

On the other hand, setting the probe beam at the plasmonic wavelength is to further ensure that the transient absorption signal of the probe beam $\left(\Delta \sigma_{\mathrm{abs}}\right)$ is linearly proportional to the temperature change of GNRs $\left(\Delta T_{\mathrm{p}}\right)$. According to Eq. (1) in the paper, this would require the first term to be sufficiently bigger than the second term in Eq. (1). The advantage of setting probe beam at the plasmonic wavelength is that the influence coefficient of $\Delta T_{\mathrm{p}}$, $B_{\mathrm{p}}=\frac{\partial \sigma_{\mathrm{abs}}}{\partial \varepsilon_{1}} \frac{d \varepsilon_{1}}{d T_{\mathrm{p}}}+\frac{\partial \sigma_{\mathrm{abs}}}{\partial \varepsilon_{2}} \frac{d \varepsilon_{2}}{d T_{\mathrm{p}}}$ is much higher than that of $\Delta T_{\mathrm{m}}, B_{\mathrm{m}}=\frac{\partial \sigma_{\mathrm{abs}}}{\partial \varepsilon_{\mathrm{m}}} \frac{d \varepsilon_{\mathrm{m}}}{d T_{\mathrm{m}}}$. To better illustrate this, we plot $B_{\mathrm{p}}$ and $B_{\mathrm{m}}$ in the wavelength range of $650 \mathrm{~nm}$ to $850 \mathrm{~nm}$ in Figure $\mathrm{S} 2$. In calculation of $B_{\mathrm{p}}$ and $B_{\mathrm{m}}$, the temperature dependent dielectric functions of the GNR and the water medium are $d \varepsilon_{1} / d T_{\mathrm{p}} \approx 2 \times 10^{-3} \mathrm{~K}^{-1}, d \varepsilon_{2} / d T_{\mathrm{p}} \approx 2 \times 10^{-3} \mathrm{~K}^{-1},{ }^{4}$ and $d \varepsilon_{\mathrm{m}} / d T_{\mathrm{m}} \approx-2 \times 10^{-4}$ $\mathrm{K}^{-1}{ }^{5-6}$ respectively. $\partial \sigma_{\mathrm{abs}} / \partial \varepsilon_{1}, \partial \sigma_{\mathrm{abs}} / \partial \varepsilon_{2}$, and $\partial \sigma_{\mathrm{abs}} / \partial \varepsilon_{\mathrm{m}}$ are derived from the Gans theory. The dielectric constants of gold are taken from Palik. ${ }^{7}$ Clearly from Figure S2, the ratio of $B_{\mathrm{p}} / B_{\mathrm{m}}$ 
reaches the maximum value of $\sim 30$ at the plasmonic wavelength. Thus, when probe beam deviates from the plasmonic wavelength, the heating of surrounding medium can bring in substantial contribution to $\Delta \sigma_{\text {abs, }}$, especially at long time delay when heat dissipates into the medium. This would lead to the assumption of $\Delta \sigma_{\mathrm{abs}}$ being linearly proportional to $\Delta T_{\mathrm{p}}$ invalid for the model analysis. Similar observations and explanations have also been presented and discussed by Park et al..$^{8-9}$

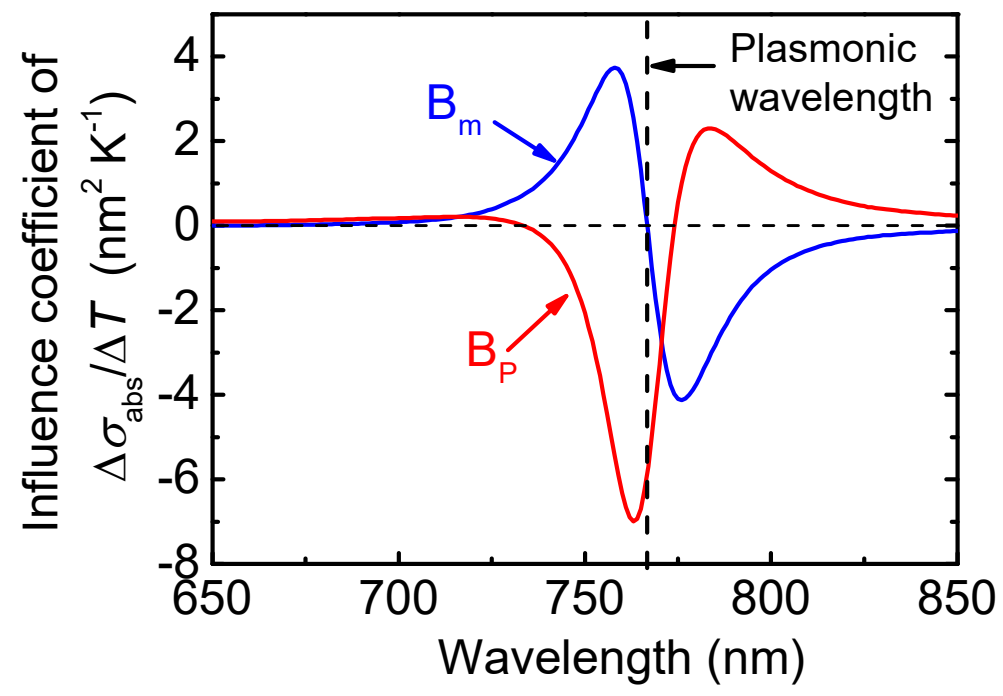

Figure S2. Absorption coefficient $B_{\mathrm{p}}$ (red line) and $B_{\mathrm{m}}$ (blue line) calculated by the Gans theory for the GNR/CTAB sample. The vertical black dash line denotes plasmonic wavelength.

\section{S4. Control Measurements on the Water Solvent}

To better illustrate the negligible contribution from the photothermal heating effect of the solvent water to the transient absorption signal, we plot the transient absorption measurement data of GNR/3K-PEG and the milli-q water in Figure S3. Both data were acquired under the same measurement conditions and were normalized by the absorption value of GNR/3K-PEG sample at $10 \mathrm{ps}$. It is clearly shown that over the time delay from $10 \mathrm{ps}$ to $2 \mathrm{~ns}$, the transient absorption signals of milli-q water are one to two orders of magnitude lower than those of the 
GNR/3K-PEG sample. Thus it is reasonable for us to neglect the photothermal heating effect of the solvent water when analyzing the transient absorption data of GNR samples.

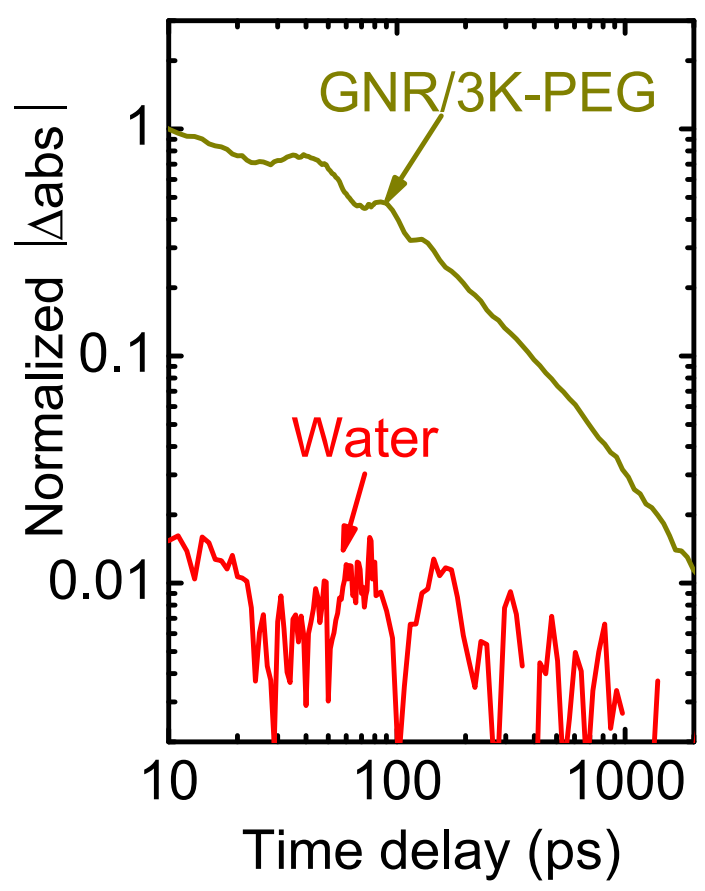

Figure S3. Transient absorption signal of GNR/3K-PEG (dark gold line) and pure water (red line) normalized by the absorption value of GNR/3K-PEG at 10 ps under the same power $(3 \mathrm{~mW})$.

\section{S5. Repeatability of Transient Absorption Measurements on Three Samples}

A laser power of $3 \mathrm{~mW}$ was applied to optically heat up the GNR sample, which was optimized to balance between a high signal-to-noise ratio and relatively low temperature rise of the GNRs. Upon heating, the transient absorption signal should be repeatable for different measurements, if the surfactant stays attached on the GNRs. For each sample (GNR/CTAB, GNR/1K-PEG, and GNR/3K-PEG), we took multiple measurements with a waiting time of $\sim 10$ minutes in between to ensure that all the transient absorption signals were repeatable. Representative results of the measurement repeatability are shown in Figure S4. Overall, the 
normalized transient absorption signals of each sample acquired at three different times overlap pretty well with each other. Slight fluctuation of the signal appears at long time delay ( $>1 \mathrm{~ns})$, which is due to the low signal-to-noise ratio when the temperature of the GNR decreases leading to small transient absorption signals. These fluctuations do not change the transient absorption decay features and thus do not affect the fitting results of surfactant thermal properties. Therefore, the possibility of surfactants detaching from the GNRs during heating is safely excluded, and we are confident about the fidelity of our measurements.
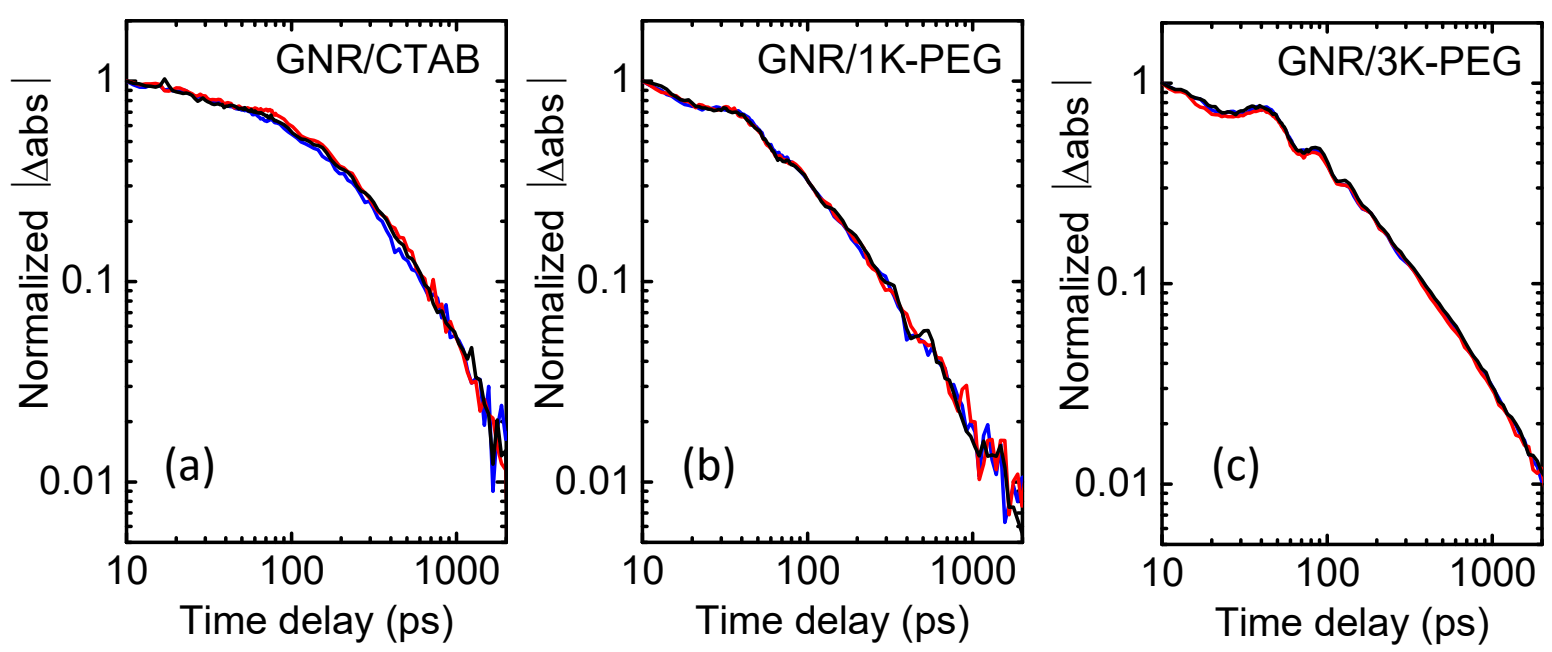

Figure S4. Repeated transient absorption measurement signals for (a) GNR/CTAB, (b) GNR/1K-PEG, and (c) GNR/3K-PEG samples. In each sub figure, blue, red, and black lines are the normalized transient absorption data for the same sample accquired at three different times with an interval time of 10 minutes.

\section{S6. Thermal Transport Model}

A heat diffusion thermal model is applied to extract the thermal properties of surfactants by fitting the transient absorption measurement data with a numerical model developed using the built-in partial differential equation toolbox of MATLAB.$^{10}$ The model solves the parabolic heat diffusion partial differential equation in cylindrical coordinates, ${ }^{10}$ 


$$
\frac{\partial}{\partial r}\left(\Lambda r \frac{\partial T}{\partial r}\right)+\Lambda r\left(\frac{\partial^{2} T}{\partial z^{2}}\right)+r \dot{q}=r C \frac{\partial T}{\partial t}
$$

where $\Lambda$ is the thermal conductivity, $C$ is the volumetric heat capacity, $r$ and $z$ denote the radial and axial directions in the cylindrical coordinates, $t$ represents time for the transient term and $\dot{q}$ is the volumetric heat source. The time history of this heat source is represented as a Gaussian pulse as defined in Eq. (S7). The pulse duration $\sigma$ in Eq. (S7) is set to be $450 \mathrm{fs}$, which is the duration of the pulse trains of the pump and probe beams at the sample surface, as characterized by two-photon absorption with a fast-response Ge detector.

$$
\dot{q}=\frac{2 Q}{\sigma \sqrt{2 \pi}} \exp \left(\frac{-t^{2}}{2 \sigma^{2}}\right) .
$$

Owing to the geometric symmetry, the thermal modeling can be simplified to a twodimensional axially symmetric heat transfer problem (with the $r$ and $z$ coordinates) and only takes a quarter of one GNR as the computational region (due to symmetry about the $r$ axis). The subareas in the computational region, from the inside to the outside, are gold, the gold-surfactant interface, surfactant, the surfactant-water interface, and the surrounding water (see Figure 3a). The heat generation term is localized in the gold region only. The time step of the simulation is 1 ps. The values for $\Lambda$ and $C$ are $310 \mathrm{~W} \mathrm{~m}^{-1} \mathrm{~K}^{-1}$ and $130 \mathrm{~J} \mathrm{~kg}^{-1} \mathrm{~K}^{-1}$ for gold, and $0.58 \mathrm{~W} \mathrm{~m}^{-1} \mathrm{~K}^{-1}$ and $4180 \mathrm{~J} \mathrm{~kg}^{-1} \mathrm{~K}^{-1}$ for water.

\section{S7. Uncertainty of Interfacial Conductance Calculations from MD Simulations}

Figure S5 shows the normalized $\Delta T$ autocorrelation function (ACF) of the Au/PEG interface averaged over 30 independent simulation runs. The ACF converged to zero at 200 ps. The 
thermal conductance was determined from the integral of the averaged ACF according to Eq. (3), up to this identified convergence time.

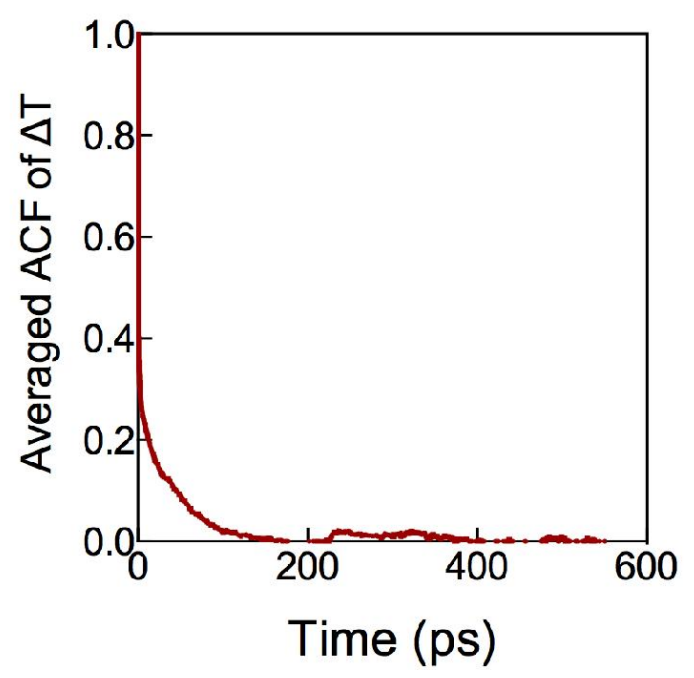

Figure S5.The normalized $\Delta T$ autocorrelation function of $\mathrm{Au} / \mathrm{PEG}$ interface averaged over 30 simulation runs.

The $\triangle T$ ACFs of $\mathrm{Au} /$ water and $\mathrm{PEG} /$ water converged more slowly due to the random and rapid movement of water molecules, especially for large simulation systems. To achieve a better $\mathrm{ACF}$ convergence, our data of $G$ of $\mathrm{Au} /$ water and $\mathrm{PEG} /$ water were calculated from a supercell with a smaller cross-sectional area $\left(A_{\mathrm{c}}\right)$ of $1.0 \times 1.1 \mathrm{~nm}$. A smaller supercell efficiently imposes a constraint on the random motion of water molecules and gives a better $\Delta T$ ACF convergence. The finite size effect is not significant in our calculations, for example, $G_{\mathrm{Au}-\mathrm{PEG}}$ of $146 \pm 22 \mathrm{MW} \mathrm{m}^{-2} \mathrm{~K}^{-1}$ calculated from $A_{\mathrm{c}}=1.0 \times 1.1 \mathrm{~nm}$ is within the error bar of that calculated from $A_{\mathrm{c}}=4.1 \times 4.1 \mathrm{~nm}\left(G_{\mathrm{Au}-\mathrm{PEG}}=163 \pm 28 \mathrm{MW} \mathrm{m}^{-2} \mathrm{~K}^{-1}\right)$.

The error bars are defined as follows: each ACF-converged independent simulation gives a $G$ value. The average $G$ as a function of simulation numbers is plotted in Figure S6 for the $\mathrm{Au} / \mathrm{PEG}$ interface as a representative example. 


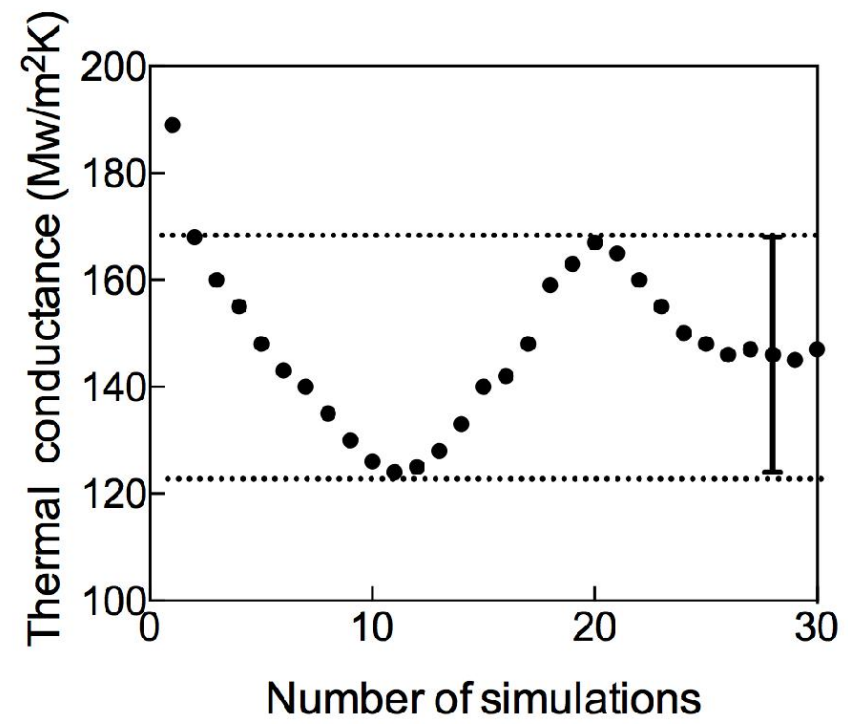

Figure S6. Averaged thermal conductance functions the number of simulations.

After a sufficient number of simulations, the average value of thermal conductivity converges to a constant value. The error bars are then defined by taking into account the variance of the averaged $G$, as seen in Figure S6.

\section{S8. Sensitivity Analysis of Transient Absorption and Uncertainty Estimation}

The in-phase signal of transient absorption measurements is proportional to the GNR temperature, and thus the sensitivity of the in-phase signal to a certain parameter essentially represents that of the GNR temperature, which is defined as:

$$
S_{\alpha}=\frac{\partial \ln (T)}{\partial \ln (\alpha)},
$$

where $\alpha$ is a parameter of interest, $S_{\alpha}$ is the sensitivity to parameter $\alpha$, and $T$ is the GNR temperature. 
Figure S7 depicts the sensitivity of the GNR temperature to the interfacial thermal conductance $\left(G_{1}\right.$ and $\left.G_{2}\right)$, the thickness $\left(h_{\mathrm{s}}\right)$, thermal conductivity $\left(\Lambda_{\mathrm{s}}\right)$, and the heat capacity $\left(C_{\mathrm{s}}\right)$ of the surfactant layer as well as thermal conductivity $\left(\Lambda_{\mathrm{m}}\right)$, and the heat capacity $\left(C_{\mathrm{m}}\right)$ of the surrounding medium (water) as functions of the time delay. Since the difference in the sensitivity analysis for model 1 and 2 is negligible, the sensitivity analysis for model 1 is not presented in Figure S7. As shown in Figure S7a, when $G_{1}$ and $G_{2}$ are sufficiently large (models 1 and 2), the transient absorption signal (or the GNR temperature) is not sensitive to either interfacial thermal conductance. However, when the smaller values of $G_{1}$ predicted from MD are used, the transient absorption signal becomes sensitive to $G_{1}$. Subsequently, the uncertainties of $G_{1}$ propagated from the MD simulation will make substantial contributions to the overall uncertainties of $\Lambda_{\mathrm{s}}$ and $C_{\mathrm{s}}$ of the surfactant layer. Over the entire time delay, the transient absorption signal is quite sensitive to $\Lambda_{\mathrm{s}}$ from a few tens of picoseconds up to $1-2 \mathrm{~ns}$, and sensitive to $C_{\mathrm{s}}$ typically at longer time delays from hundreds of picoseconds to nanoseconds. 

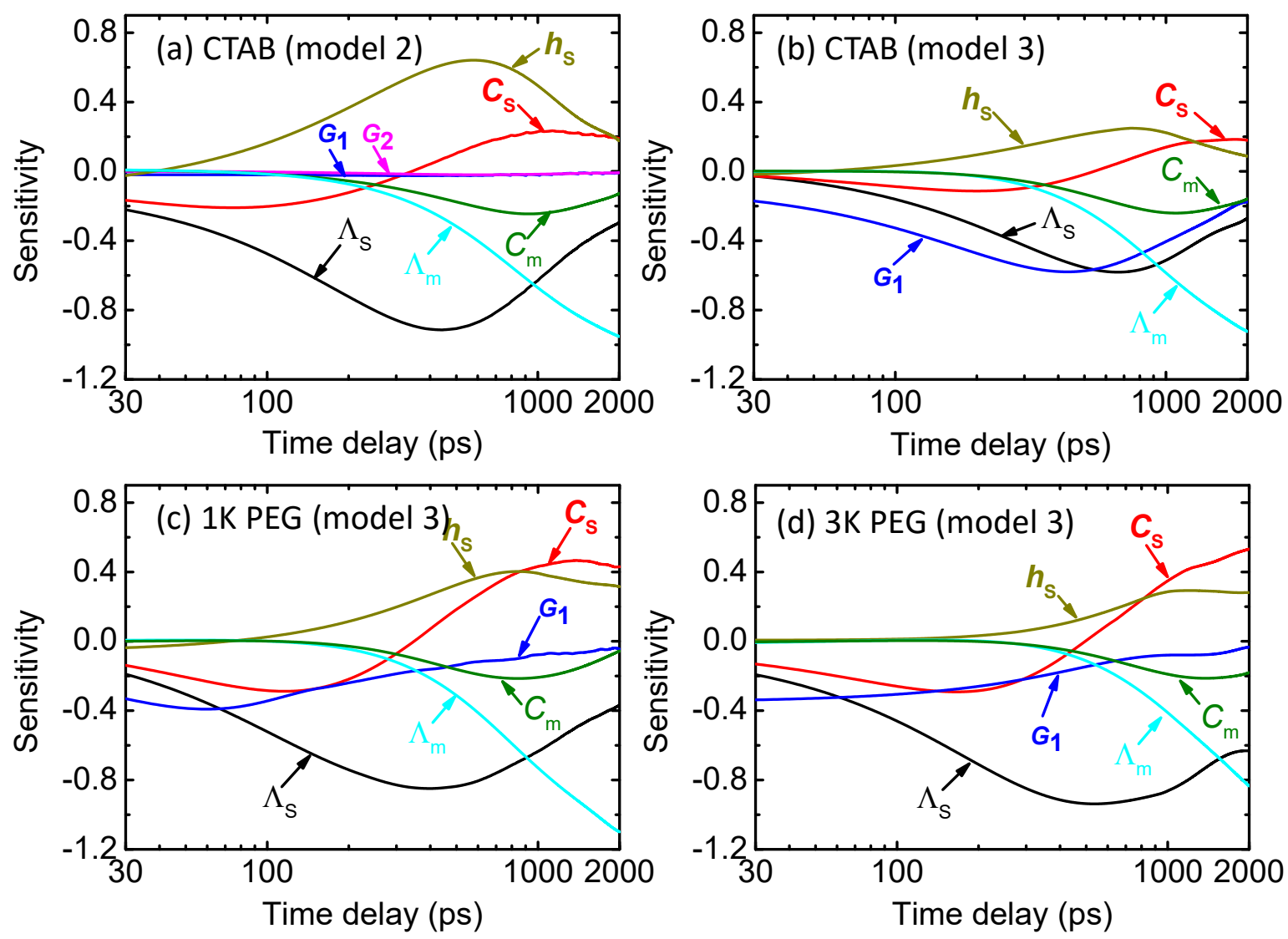

Figure S7. Sensitivity analysis of the GNR temperature to different parameters as functions of the time delay: (a) Sample 1 (CTAB) with $G_{1}=G_{2}=1 \mathrm{GW} \mathrm{m}^{-2} \mathrm{~K}^{-1}$ (model 2); (b) Sample 1 (CTAB) of model 3 with $G_{1}=83 \mathrm{MW} \mathrm{m}^{-2} \mathrm{~K}^{-1}$ predicted from MD (model 3); (c) Sample 2 ( $1 \mathrm{~K}$ PEG) of model 3; (d) Sample 3 (3K PEG) of model 3. For both (c) and (d), $G_{1}$ $=230 \mathrm{MW} \mathrm{m}^{-2} \mathrm{~K}^{-1}$ as predicted from MD for Au/PEG. The surfactant thicknesses are $4.7 \mathrm{~nm}$ for CTAB in both (a) and (b), $5.4 \mathrm{~nm}$ for $1 \mathrm{~K}$ PEG in (c), and $9.0 \mathrm{~nm}$ for $3 \mathrm{~K}$ PEG in (d).

The uncertainties of the thermal conductivity and heat capacity of the surfactant layer can be estimated by taking into account individual uncertainties based on the error propagation formula:

$$
\left(\frac{\Delta \Lambda_{\mathrm{s}}}{\Lambda_{\mathrm{s}}}\right)^{2}=\sum\left(\frac{S_{\alpha}}{S_{\Lambda_{\mathrm{s}}}} \frac{\Delta \alpha}{\alpha}\right)^{2}
$$


where $\Delta \Lambda_{\mathrm{s}} / \Lambda_{\mathrm{s}}$ is the uncertainty of measured $\Lambda_{\mathrm{s}}, \Delta \alpha / \alpha$ is the uncertainty of parameter $\alpha, S_{\alpha}$ is the sensitivity to parameter $\alpha$, and $S_{\Lambda \mathrm{s}}$ is the sensitivity to $\Lambda_{\mathrm{s}}$. $\Lambda_{\mathrm{s}}$ in Eq. (S9) can be replaced by $C_{\mathrm{s}}$ for calculating the uncertainty of $C_{\mathrm{s}}$.

The dominant parameters contributing to the overall uncertainties of $\Lambda_{\mathrm{s}}$ and $C_{\mathrm{s}}$ are $h_{\mathrm{s}}$ and the $G_{1}$ value from MD simulation. The uncertainty of $h_{\mathrm{s}}$ is estimated based on Eq. (S9) with $L_{\mathrm{GNR}}$, $d_{\mathrm{GNR}}$, and $d_{\mathrm{H}}$ being the individual parameters of $\alpha$. The uncertainties of $L_{\mathrm{GNR}}$ and $d_{\mathrm{GNR}}$ are evaluated based on the standard deviation of the GNR size distribution collected from TEM images (Table 1). The uncertainty of $d_{\mathrm{H}}$ is estimated by the standard error of the Gaussian fit to the DLS measurements (Table 1). The final uncertainties of surfactant thickness are $20 \%, 17 \%$, $11 \%$ for CTAB, $1 \mathrm{~K}$ PEG, and $3 \mathrm{~K}$ PEG samples respectively. Together with the uncertainty of $G_{1}$ from the MD simulation, the overall uncertainties are from $8 \%$ to $17 \%$ for $\Lambda_{\mathrm{s}}$, from $15 \%$ to $38 \%$ for $C_{\mathrm{s}}$, and from $18 \%$ to $25 \%$ for $G_{\text {eff }}$ as shown in Table 3 .

\section{S9.Comparison of $G_{\text {eff }}$ in the Cylindrical and Cartesian Coordinates}

The shape of a GNR can be represented by a circular cylinder capped with two hemispheres on both ends. In this work, cylindrical coordinates are used to estimate $G_{\text {eff }}$ using the thermal resistance network as described by Eq. (2). The cylindrical coordinates for solving the heat diffusion governing equation have taken into account the surface area variation along the radial direction of surfactant layers. In addition, the calculation of $G_{\text {eff }}$ in Eq. (2) is also derived in cylindrical coordinates, such that radial dependence of the surface area is automatically incorporated in the modeling. We noticed that in previous works $G_{\text {eff }}$ was calculated in Cartesian coordinates, as expressed in Eq. (S10): ${ }^{9,11}$ 


$$
\frac{1}{G_{\text {eff }}}=\frac{1}{G_{1}}+\frac{1}{G_{\mathrm{s}}}+\frac{1}{G_{2}} .
$$

The comparison of $G_{\text {eff }}$ for all three samples calculated in two coordinate systems is summarized in Figure S8. It is can be seen from Figure S8 that for models 1 and 2 with large values of $G_{1}$, the deviation of $G_{\text {eff }}$ calculated in both coordinate systems is small (less than $10 \%$ ). However, for model 3 with smaller $G_{1}$ from the MD simulation, the values of $G_{\text {eff }}$ calculated in the Cartesian coordinates (black symbols) are nearly $20 \%$ higher than those calculated in the cylindrical coordinates (red symbols). Nevertheless, the derivation of $G_{\text {eff }}$ due to the coordinate selection is within the overall uncertainty of $G_{\text {eff. }}$

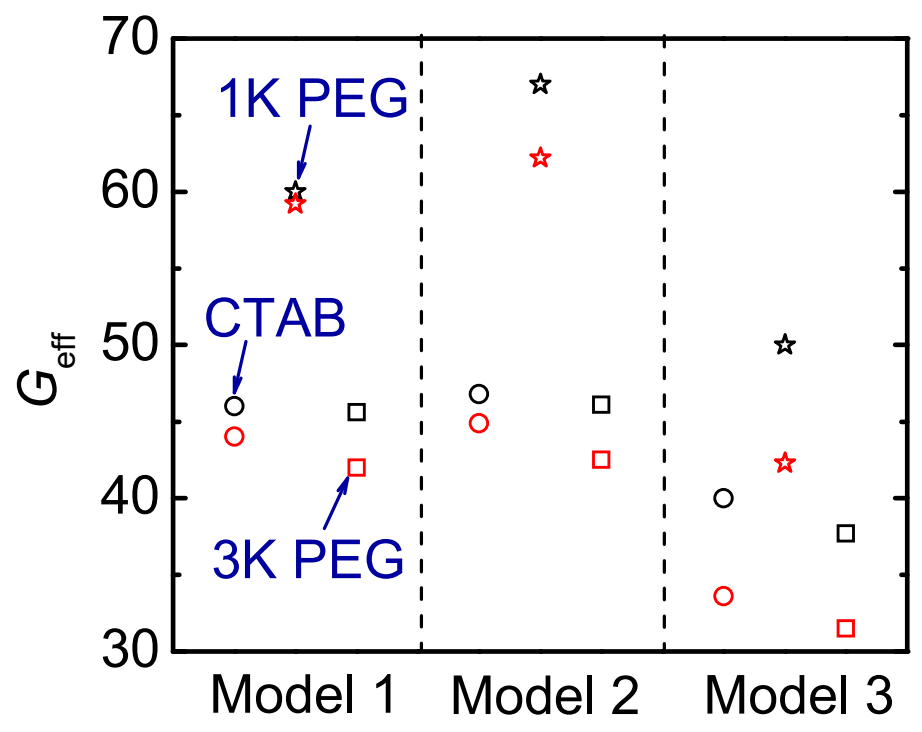

Figure S8. $G_{\text {eff }}$ calculated in three models with cylindrical (red symbols) and Cartesian (black symbols) coordinates for all three samples (CTAB - open circles, 1K PEG - stars, and 3K PEG open squares). The deviations of $G_{\text {eff }}$ calculated in the cylindrical and Cartesian coordinates range from $1 \%$ to $8 \%$ for model $1,4 \%$ to $8 \%$ for model 2 , and $18 \%$ to $19 \%$ for model 3 with smaller $G_{1}$ predicted from the MD simulations. 


\section{S10. Discussion of Parameters that Influence the Transient Temperature Decay Process}

From Eq. (S6), the transient temperature decay in the GNR depends on the thermal conductivity $\Lambda$, the volumetric heat capacity $C$, and the heat source $\dot{q}$ in the model. According to the fitting results in Table 3, the $G_{\text {eff }}$ of model 3 decreases by $30-40 \%$ compared with $G_{\text {eff }}$ of model 1. To further support this result, we present simulated transient temperature decay in the GNR with model 3, in which we still use $G_{1}$ calculated from MD but with the imposed $G_{\text {eff }}$ of model 1, i.e. with $\Lambda_{\mathrm{s}}$ extracted from equation (2). We use $C_{\mathrm{s}}$ of the surfactant obtained from model 1. Detailed input parameters are summarized in Table S1. The simulation plots depicted in Figure S9 demonstrate distinct decay curves for the two models and in both samples.

Table S1. Input of the thermal properties for the simulation of GNR/surfactant/water structure.

\begin{tabular}{|c|c|c|c|c|c|c|}
\hline \multirow{2}{*}{$\begin{array}{c}\text { Sample } \\
(\#)\end{array}$} & \multirow{2}{*}{ Model (\#) \& Structure } & $G_{1}$ & $G_{2}$ & $G_{\text {eff }}$ & \multirow{2}{*}{$\frac{\Lambda_{\mathrm{s}}}{\left(\mathrm{W} \mathrm{m}^{-1} \mathrm{~K}^{-1}\right)}$} & \multirow{2}{*}{$\frac{C_{\mathrm{s}}}{\left(\mathrm{J} \mathrm{cm}^{-3} \mathrm{~K}^{-1}\right)}$} \\
\hline & & \multicolumn{3}{|c|}{$\left(\mathrm{MW} \mathrm{m}^{-2} \mathrm{~K}^{-1}\right)$} & & \\
\hline \multirow{2}{*}{ A } & 1. $\mathrm{Au} / \mathrm{CTAB} /$ water & - & - & 45 & 0.22 & 1.6 \\
\hline & 3. $\mathrm{Au} / G_{1}(\mathrm{MD}) / \mathrm{CTAB} / G_{2} /$ water & 83 & $>10^{4}$ & 45 & 0.76 & 1.6 \\
\hline \multirow{2}{*}{ B } & 1. $\mathrm{Au} / 1 \mathrm{~K}-\mathrm{PEG} /$ water & - & - & 62 & 0.34 & 3.0 \\
\hline & 3. $\mathrm{Au} / G_{1}(\mathrm{MD}) / 1 \mathrm{~K}-\mathrm{PEG} / G_{2} /$ water & 230 & $>10^{4}$ & 62 & 0.62 & 3.0 \\
\hline
\end{tabular}
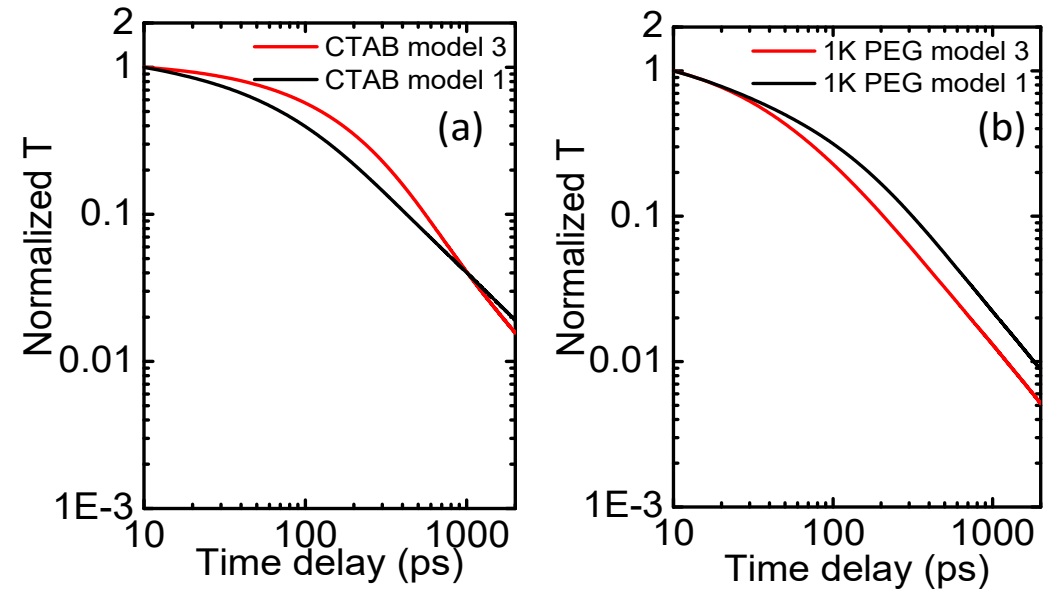

Figure S9. Simulation of transient temperature decay in the GNR for model 1 and model 3 with the same $G_{\text {eff }}$ and $C_{\mathrm{s}}$ for the surfactant layer: (a) CTAB and (b) $1 \mathrm{~K}$ PEG. 
In the steady-state situation, the total thermal resistance is the only factor affecting the heat transfer rate. In transient thermal transport the heat capacity is as important as the thermal resistance and it also determines the heat transfer rate at different locations. In model 3, there is a temperature drop at the GNR/surfactant interface since $G_{1}$ is not infinitely large, but no thermal energy could accumulate at the interface because its heat capacity is zero. At the same time, the rest of the gold and surfactant have both a temperature gradient and an energy accumulation caused by the heat flux gradient simultaneously. Conversely, in model 1 without the interfacial thermal resistance, this kind of temperature gradient and energy accumulation occur consistently and uniformly within the whole domain, making the temperature decay different from that of model 3 .

\section{S11. Effect of $G_{2}$ on the Fitting Results}

As discussed in the paper (Section 4.2) in model 3, $G_{2}$ (polymer: water interface) is set to be large enough $\left(>10^{4} \mathrm{MW}^{-1} \mathrm{~m}^{-2} \mathrm{~K}^{-1}\right)$ for three samples during the fitting process, of which the value will not affect the fitting results. In this case, the effect of interface 2 (surfactant/water) to the thermal transport process is lumped into the thermal transport of the surfactant layer. The fitting results of model 1 and model 2 (Table 3 in the paper) for each sample have demonstrated that the interfacial thermal conductance has no influence on the fitted thermal properties of surfactant layer when it is set to be large enough $\left(>10^{4} \mathrm{MW}^{-1} \mathrm{~m}^{-2} \mathrm{~K}^{-1}\right)$. We have also tried fitting with $G_{2}$ in a range of high values from $1000 \mathrm{MW}^{-1} \mathrm{~m}^{-2} \mathrm{~K}^{-1}$ to $10^{4} \mathrm{MW}^{-1} \mathrm{~m}^{-2} \mathrm{~K}^{-1}$ in model 3, the fitted thermal conductivity and heat capacity of surfactant in each sample showed no difference in this range. 


\section{References}

(1) Orendorff, C. J.; Murphy, C. J. Quantitation of Metal Content in the Silver-Assisted Growth of Gold Nanorods. J. Phys. Chem. B 2006, 110 (9), 3990-3994.

(2) Rodríguez-Fernández, J.; Perez-Juste, J.; Liz-Marzan, L. M.; Lang, P. R. Dynamic Light Scattering of Short Au Rods with Low Aspect Ratios. J. Phys. Chem. C 2007, 111 (13), 50205025.

(3) Ortega, A.; de la Torre, J. G. Hydrodynamic Properties of Rodlike and Disklike Particles in Dilute Solution. J. Chem. Phys 2003, 119 (18), 9914-9919.

(4) Winsemius, P.; Van Kampen, F.; Lengkeek, H.; Van Went, C. Temperature Dependence of the Optical Properties of Au, Ag and Cu. J. Phys. Met. Phys. 1976, 6 (8), 1583-1606.

(5) Bashkatov, A. N.; Genina, E. A. In Water Refractive Index in Dependence on Temperature and Wavelength: A Simple Approximation, Saratov Fall Meeting 2002: Optical Technologies in Biophysics and Medicine IV, 2003; International Society for Optics and Photonics, pp 393-395.

(6) Haynes, W. M. CRC Handbook of Chemistry and Physics; CRC press2014.

(7) Palik, E. D. Handbook of Optical Constants of Solids; Academic press1998; Vol. 3.

(8) Park, J.; Cahill, D. G. Plasmonic Sensing of Heat Transport at Solid-Liquid Interfaces. $J$. Phys. Chem. C 2016, 120 (5), 2814-2821.

(9) Park, J.; Huang, J. Y.; Wang, W.; Murphy, C. J.; Cahill, D. G. Heat Transport between Au Nanorods, Surrounding Liquids, and Solid Supports. J. Phys. Chem. C 2012, 116 (50), 2633526341.

(10) Schmidt, A. J.; Alper, J. D.; Chiesa, M.; Chen, G.; Das, S. K.; Hamad-Schifferli, K. Probing the Gold Nanorod- Ligand- Solvent Interface by Plasmonic Absorption and Thermal Decay. J. Phys. Chem. Lett. 2008, 112, 13320-13323.

(11) Huang, J.; Park, J.; Wang, W.; Murphy, C. J.; Cahill, D. G. Ultrafast Thermal Analysis of Surface Functionalized Gold Nanorods in Aqueous Solution. ACS Nano 2012, 7 (1), 589-597. 